\title{
Electrocatalysis and Determination of Ascorbic Acid Through Graphite Paste Electrode Modified With Iron Nitroprusside
}

\author{
Devaney R. do Carmo, Ricardo M. da Silva, Nelson R. Stradiotto* \\ Instituto de Química, Universidade Estadual Paulista \\ (UNESP) CEP 14800-900 Araraquara, SP - Brasil.
}

Received 25 October 2002; accepted in revised form 9 January 2004

\begin{abstract}
Iron nitroprusside, $\mathrm{Fe}(\mathrm{II}) \mathrm{NP}$ was incorporated into a carbon paste electrode and the electrochemical studies were performed by cyclic voltammetry. The cyclic voltammogram of the modified electrode, $\mathrm{Fe}(\mathrm{II}) \mathrm{NP}$ showed two redox couples $\left(\mathrm{E}_{1 / 2}{ }^{\mathrm{ox}}\right)_{1}=$ 0.24 and $\left(\mathrm{E}_{1 / 2}{ }^{\mathrm{ox}}\right)_{2}=0.85 \mathrm{~V}$ vs. SCE attributed to $\mathrm{Fe}^{(\mathrm{II})} / \mathrm{Fe}^{(\mathrm{III})}$ and $\mathrm{Fe}^{(\mathrm{II})}(\mathrm{CN})_{5} \mathrm{NO} /$ $\mathrm{Fe}^{(\mathrm{III})}(\mathrm{CN})_{5} \mathrm{NO}$, respectively. The redox couple at $\left(\mathrm{E}_{1 / 2}{ }^{\mathrm{ox}}\right)_{1}=0.24 \mathrm{~V}$ presents an electrocatalytic response for ascorbic acid. The modified paste electrode exhibits a decrease of $180 \mathrm{mV}$ in the ascorbic acid oxidation. The modified graphite paste electrode gives a linear range from $1.0 \times 10^{-3}$ to $2.6 \times 10^{-2} \mathrm{~mol} \mathrm{~L}^{-1}$ of ascorbic acid with a detection limit of $1.4 \times 10^{-3}( \pm 1.2 \%) \mathrm{mol} \mathrm{L}^{-1}(\mathrm{n}=3)$. The electrocatalytic oxidation of ascorbic acid compounds by the mediator has been used for the determination of ascorbic acid in a commercially pharmaceutical available product.
\end{abstract}

Keywords: iron nitroprusside, modified electrode, electrocatalysis, ascorbic acid oxidation.

\section{Introdução}

$\mathrm{Na}$ área de eletroquímica demonstra-se que eletrodos quimicamente modificados possuem algumas vantagens sobre eletrodos convencionais, contribuindo no desenvolvimento de inúmeras aplicações eletroanalíticas, dentre os quais se incluem eletrocatálise e sensores eletroquímicos [1-7].

As propriedades dos eletrodos modificados dependem da formação e das características do filme formado na superfície do eletrodo. Uma das principais

\footnotetext{
* Corresponding author. E-mail address: nrstradi@iq.unesp.br
} 
vantagens dos eletrodos quimicamente modificados é a sua capacidade de catalisar a oxidação de algumas espécies que exibem elevado potencial em eletrodos não modificados, aumentando assim sua sensibilidade e seletividade [1,8-9].

Particularmente filmes de nitroprussiato de metais de transição $\mathrm{Cu}(\mathrm{II}, \mathrm{Ni}(\mathrm{II})$, Co(II)) são de difícil preparação o que explica o pequeno número de trabalhos existente na literatura. Muitos problemas surgem na sua utilização, tais como, pequena estabilidade eletroquímica e principalmente falta de reprodutibilidade na sua formação, sendo este último assunto motivo de grandes controvérsias [10]. Visando estabelecer uma alternativa de metodologia, este trabalho sugere a exploração destes complexos insolúveis empregando eletrodos de pasta de carbono, pelo fato de ter uma maior reprodutibilidade, facilidade e rapidez de síntese sem alterar as propriedades eletrocatalíticas desta interessante classe de compostos. Recentemente descreveu-se a síntese e caracterização do nitroprussiato de ferro (Fe(II)NP) [11]. Como continuidade deste estudo descrevemos neste trabalho as capacidades eletrocatalíticas do eletrodo de pasta de grafite modificada com aquele complexo, como mediador na oxidação do acido ascórbico.

O ácido ascórbico desperta muito interesse devido aos efeitos benéficos como vitamina, além de seu uso como reagente e aditivo em alimentos. A literatura está repleta de diferentes métodos para determinação de ácido ascórbico em diferentes matrizes [12]. Destes métodos destacam-se os métodos colorimétricos [13], fluorométricos [14], potenciométricos [15], quimiluminescentes [16], e cromatográficos [17-18]. Os métodos voltamétricos empregados para a determinação de vitamina $\mathrm{C}$ descritos na literatura utilizam eletrodos convencionais [19] e eletrodos de pasta de carbono [20], porém a aplicabilidade destes eletrodos decresce com os repetitivos usos devido aos danos causados pelos produtos resultantes da oxidação [12]. Considerando as excelentes características eletroquímicas apresentadas pelo $\mathrm{Fe}(\mathrm{II}) \mathrm{NP}$, tais como, reprodutibilidade e estabilidade, pretende-se explorar neste trabalho a aplicação 
direta das propriedades eletrocatalícas de Fe(II)NP na determinação do acido ascórbico num fármaco.

\section{Método Experimental}

Todos os reagentes utilizados foram de pureza analítica (p.a Merk), sendo a água deionizada através do Milli-Q Gradiente da Millipore.

A quantificação de $\mathrm{Fe}$ foi feita por espectroscopia de absorção atômica empregando-se um espectrômetro Perkin Elmer, modelo 300. O teor de Fe no sólido formado foi determinado por absorção atômica sendo obtido o valor de $(36.0 \%)$, calculado $(36.3 \%)$.

A caracterização do complexo na região do infravermelho foi feita em pastilha de $\mathrm{KBr}$ empregando um espectrofotômetro Infravermelho Nicolet 730 SX-FT, entre 4000 e $400 \mathrm{~cm}^{-1}$. O infravermelho de Fe(II)NP apresentou os seguintes modos vibracionais: $\mathrm{CN}_{\mathrm{str}}\left(2182 \mathrm{~cm}^{-1}\right)$; $\mathrm{NO}_{\text {str }}\left(1945 \mathrm{~cm}^{-1}\right)$ e $\mathrm{Fe}-\mathrm{NO}_{\text {bend }}\left(647 \mathrm{~cm}^{-1}\right)$, os quais estão em concordância com os resultados encontrados na literatura [11].

Para as medidas de voltametria cíclica empregou-se um potenciostato /galvanostato da AutoLab (PGSTAT 30). O sistema eletroquímico empregado foi constituído de três eletrodos: o eletrodo auxiliar de platina, o eletrodo de referência de calomelano saturado (ECS) e o eletrodo de trabalho de pasta de grafite. O eletrodo de trabalho consiste simplesmente de um tubo de vidro de 15 cm de comprimento e diâmetro interno de $0.30 \mathrm{~cm}$ e diâmetro externo de $0.5 \mathrm{~cm}$ sendo cavidade interna conectada por um fio de cobre para estabelecer contato elétrico. A pasta de grafite modificada com Fe (II)NP foi preparada misturando $10 \mathrm{mg}$ de $\mathrm{Fe}(\mathrm{II}) \mathrm{NP}$ com $90 \mathrm{mg}$ de grafite em pó (Aldrich) e $50 \mu \mathrm{L}$ de óleo mineral.

Voltamogramas cíclicos foram registrados para o estudo da eletrocatálise do ácido ascórbico. As soluções de ácido ascórbico foram preparadas imediatamente antes do uso e as soluções foram purgadas com nitrogênio antes de cada medida. $\mathrm{Na}$ maioria dos experimentos empregou-se como eletrólito suporte $1.0 \mathrm{M} \mathrm{KCl}$ $(\mathrm{pH} \sim 3.0)$. A corrente catalítica foi estimada pela diferença entre a corrente medida na presença de acido ascórbico e aquela estabelecida na sua ausência. As 
soluções foram desaeradas com nitrogênio durante 10 minutos antes da realização das medidas. Os voltamogramas cíclicos foram registrados em soluções de $\mathrm{KCl} 1.0 \mathrm{~mol} \mathrm{~L}^{-1}$ (pH 3.5) na velocidade de varredura de $20 \mathrm{mV} . \mathrm{s}^{-1}$. As amostras foram determinadas empregando o método de adição padrão. $\mathrm{O}$ procedimento básico consiste em adicionar a uma cela eletroquímica contendo 50 $\mathrm{mL}$ de $\mathrm{KCl} 1.0 \mathrm{~mol} \mathrm{~L}^{-1}$ (pH 3) uma alíquota da amostra (Cewin) (50 $\left.\mu \mathrm{L}\right)$, em seguida duas alíquotas $(100$ e $200 \mu \mathrm{L})$ de solução padrão de ácido ascórbico (1.0 mol L $\left.{ }^{-1}\right)$.

\section{Resultados e Discussão}

\section{Eletrocatálise}

O comportamento eletroquímico do eletrodo de pasta de carbono modificado com $\mathrm{Fe}(\mathrm{II}) \mathrm{NP}$, descrito anteriormente [10], utilizando a técnica de voltametria cíclica, caracterizou-se por apresentar dois pares redox com potenciais médios $\left(\mathrm{E}_{1 / 2}^{\mathrm{ox}}\right)$ de 0.24 e $0.85 \mathrm{~V}\left(\mathrm{v}=20 \mathrm{mV}^{-1} \mathrm{~s}^{-1}\right.$ vs. ECS $)$ atribuídos a $\mathrm{Fe}^{(\mathrm{II})} / \mathrm{Fe}^{(\mathrm{III})} \mathrm{e}$ $\mathrm{Fe}^{(\mathrm{II})}(\mathrm{CN})_{5} \mathrm{NO} / \mathrm{Fe}^{(\mathrm{III})}(\mathrm{CN})_{5} \mathrm{NO}$, respectivamente, conforme ilustra a Fig. 1.

$$
\begin{gathered}
\mathrm{C}_{2}\left\{\mathrm{Fe}^{\mathrm{II}}\left[\mathrm{Fe}^{\mathrm{II}}(\mathrm{CN})_{5} \mathrm{NO}\right]\right\} \rightleftarrows \mathrm{C}^{+}+\mathrm{e}^{-}+\mathrm{C}\left\{\mathrm{Fe}^{\mathrm{III}}\left[\mathrm{Fe}^{\mathrm{II}}(\mathrm{CN})_{5} \mathrm{NO}\right]\right\} \\
\left(\mathrm{E}_{1 / 2}^{\mathrm{ox}}\right)_{1}=0,24 \mathrm{~V} \\
\mathrm{C}\left\{\mathrm{Fe}^{\mathrm{III}}\left[\mathrm{Fe}^{\mathrm{II}}(\mathrm{CN})_{5} \mathrm{NO}\right]\right\} \rightleftarrows \mathrm{C}^{+}+\mathrm{e}^{-}+\left\{\mathrm{Fe}^{\mathrm{III}}\left[\mathrm{Fe}^{\mathrm{III}}(\mathrm{CN})_{5} \mathrm{NO}\right]\right\} \\
\left(\mathrm{E}_{1 / 2}{ }^{\mathrm{ox}}\right)_{2}=0,85 \mathrm{~V}
\end{gathered}
$$

onde $\mathrm{C}: \mathrm{Li}^{+}, \mathrm{Na}^{+}, \mathrm{K}^{+}$.

O comportamento do eletrodo de pasta de grafite com Fe(II)NP, por analogia ao estudo com azul da Prússia [11], pode ser expresso pelas eqs. (1) e (2).

O voltamograma cíclico de pasta de grafite modificado com Fe (II)NP apresenta uma boa estabilidade eletroquímica. Durante sucessivas ciclagens a corrente anódica e catódica permanecem praticamente constantes. O eletrodo modificado com $\mathrm{Fe}(\mathrm{II}) \mathrm{NP}$ após 40 dias mergulhado no eletrólito ( $\mathrm{KCl} 1.0 \mathrm{M})$ apresenta uma pequena diminuição da corrente anódica e catódica $(\sim 9 \%)$. Estas condições tornam propício o uso deste eletrodo em eletrocatálise. 


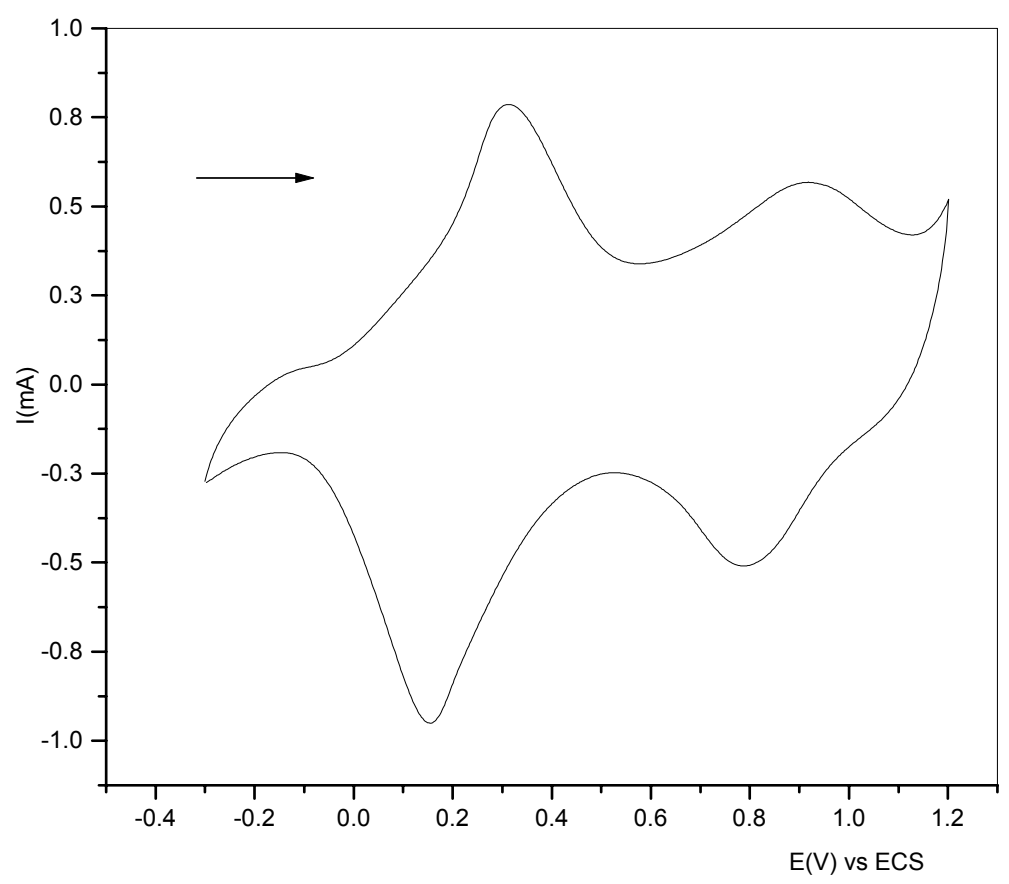

Figura 1. Voltamograma cíclico da pasta de grafite modificada com $\mathrm{Fe}(\mathrm{II}) \mathrm{NP}$ (KCl $1.0 \mathrm{M} ; \mathrm{v}=20 \mathrm{mV} \cdot \mathrm{s}^{-1}$ )

A Fig. 2 mostra o voltamograma cíclico do eletrodo de pasta de carbono sem (Fig. 2a) e com modificador (Fig. 2c). Quando se adiciona $4.8 \times 10^{-3} \mathrm{M}$ de ácido ascórbico a corrente anódica do par redox com potencial médio $\left(\mathrm{E}_{1 / 2}{ }^{\text {ox }}\right)_{1}$ de 0.24 $\mathrm{V}$ aumenta proporcionalmente à concentração de ácido ascórbico. O Fe(III) produzido durante a varredura anódica, "quimicamente" oxida o ácido ascórbico, enquanto que o Fe (III) é reduzido a Fe(II), que deverá ser outra vez oxidado a Fe(III) na superfície do eletrodo (Fig. 2d). O potencial de oxidação de ácido ascórbico em eletrodo de pasta de grafite ocorre a $0.49 \mathrm{~V}$ ( Fig. 2b).

Desta forma o ácido ascórbico é eletrocataliticamente oxidado na superfície do eletrodo. O potencial de oxidação ocorre a $0.31 \mathrm{~V}$ vs. ECS, apresentando uma diminuição de potencial de $180 \mathrm{mV}$. O processo eletrocatalítico pode ser representado pelas equações 3 e 4 .

$$
\left\{\mathrm{Fe}^{\mathrm{II}}\left[\mathrm{Fe}^{\mathrm{II}}(\mathrm{CN})_{5} \mathrm{NO}\right]\right\}-\mathrm{e}^{-} \rightleftarrows\left\{\mathrm{Fe}^{\mathrm{III}}\left[\mathrm{Fe}^{\mathrm{II}}(\mathrm{CN})_{5} \mathrm{NO}\right]\right\} \quad(0,24 \mathrm{~V})
$$




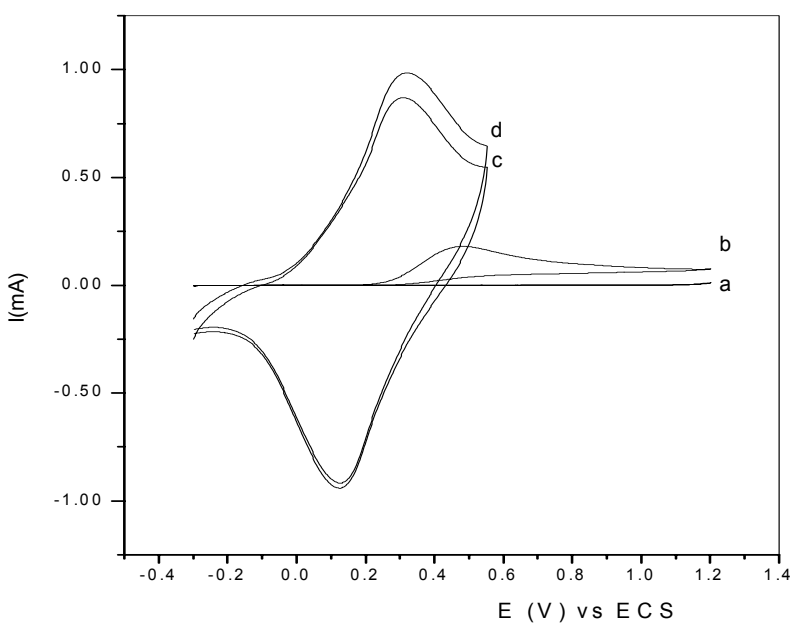

Figura 2. Voltamograma cíclico de : a ) eletrodo de pasta de grafite; b) eletrodo de pasta de grafite com adição de ácido ascórbico $(0.0048$ mol.L-1); c) eletrodo pasta de grafite modificado com $\mathrm{Fe}(\mathrm{II}) \mathrm{NP}(5 \% \mathrm{~m} / \mathrm{m})$; d) eletrodo pasta de grafite modificado com $\mathrm{Fe}(\mathrm{II}) \mathrm{NP}(5 \% \mathrm{~m} / \mathrm{m})$ adicionando-se ácido ascórbico $\left(0.0048 \mathrm{~mol} \mathrm{~L}^{-1}\right) \quad(\mathrm{v}=20$ $\left.\mathrm{mV} \cdot \mathrm{s}^{-1}\right)$.

$$
\left\{\mathrm{Fe}^{\mathrm{III}}\left[\mathrm{Fe}^{\mathrm{II}}(\mathrm{CN})_{5} \mathrm{NO}\right]\right\}+\mathrm{AA}^{-} \rightleftarrows\left\{\mathrm{Fe}^{\mathrm{II}}\left[\mathrm{Fe}^{\mathrm{II}}(\mathrm{CN})_{5} \mathrm{NO}\right]\right\}+\mathrm{DAA}^{-}+\mathrm{H}^{+}
$$

onde $\mathrm{AA}^{-}$e $\mathrm{DAA}^{-}$correspondem às formas dissociadas do ácido ascórbico e do ácido deshidroascórbico, respectivamente.

\section{Determinação}

A curva de calibração do eletrodo representada pela Fig. 3 apresenta uma faixa linear de $1.0 \times 10^{-3}$ a $2.6 \times 10^{-2} \mathrm{~mol} \mathrm{~L}^{-1}$, com uma equação linear correspondente a $\mathrm{Y}(\mathrm{mV})=17.37+18.003$ [ácido ascórbico] com um coeficiente de correlação de 0.9988 e um desvio padrão de $1.2 \%(n=3)$.

A determinação do ácido ascórbico utilizando este eletrodo de pasta de grafite modificado apresenta um limite de detecção de $1.4 \times 10^{-3} \mathrm{~mol} \mathrm{~L}^{-1}$ e uma sensibilidade amperométrica de $18 \mathrm{~mA} / \mathrm{mol} \mathrm{L}^{-1}$.

A aplicação do eletrodo de pasta de grafite modificada com $\mathrm{Fe}$ (II)NP foi testada na determinação de ácido ascórbico em um fármaco: Cewin. A Fig. 4 apresenta o comportamento voltamétrico do par redox $(0.24 \mathrm{~V})$ nas sucessivas adições de padrão do ácido ascórbico. 


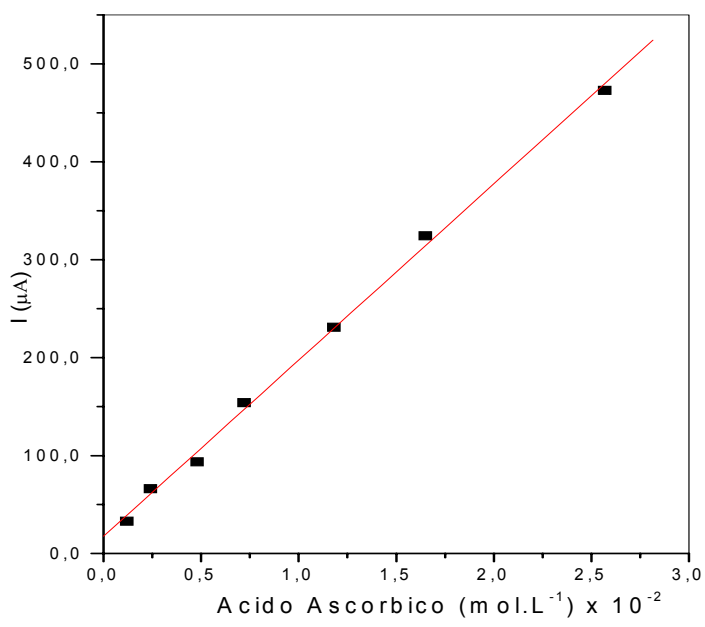

Figura 3. Curva de calibração do ácido ascórbico empregando o eletrodo de pasta de grafite modificado com $\mathrm{Fe}(\mathrm{II}) \mathrm{NP}(5 \% \mathrm{~m} / \mathrm{m})\left(\mathrm{KCl} 1.0\right.$ mol. $\mathrm{L}^{-1}$, pH 3.5).

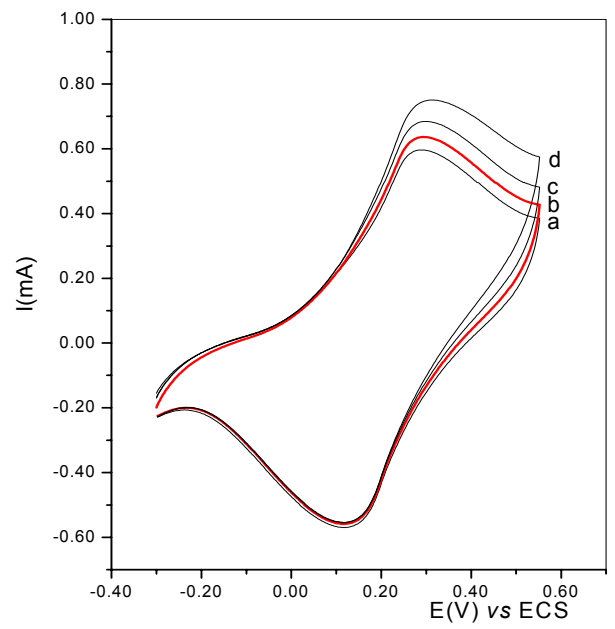

Figura 4. Voltamograma cíclico de: a) eletrodo de pasta de grafite modificado com Fe(II)NP (5\% m/m); b) com adição de $0.05 \mathrm{~mL}$ de Cewin; c) com adição de $0.1 \mathrm{~mL}$ de padrão; d) com adição de $0.2 \mathrm{~mL}$ de padrão ( concentração do acido ascórbico 0.1 $\left.\mathrm{mol} \mathrm{L}{ }^{-1}\right)\left(\mathrm{V}=20 \mathrm{mv}^{-1} \mathrm{~s}^{-1} \mathrm{KCl} 1 \mathrm{~mol} \mathrm{~L}^{-1} \mathrm{pH} 3.5\right)$.

Com o objetivo de se fazer um estudo comparativo empregou-se o método volumétrico [21], baseado na titulação com iodato de potássio. A Tabela 1 apresenta os resultados da determinação do fármaco (Cewin) utilizando o método proposto e o método preconizado na literatura [21]. 
Tabela 1. Determinação de ácido ascórbico em fármaco*

\begin{tabular}{lcc}
\hline Fármaco & Método proposto $(\mathrm{mg} / \mathrm{mL})$ & Método volumétrico $(\mathrm{mg} / \mathrm{mL})$ \\
\hline Cewin $^{* *}$ & $193.74 \pm 0.01$ & $197.42 \pm 0.00$ \\
\hline
\end{tabular}

*Resultados descritos para determinações em triplicata, ${ }^{* *}$ Solução oral, contendo: sorbitol, metilparabeno, hidróxido de sódio, sacarose, riboflavina 5- fosfato, álcool etílico, aroma artificial de caramelo. Quantidade de ácido ascórbico declarada $(\mathrm{mg} / \mathrm{mL})$ para Cewin : $200 \mathrm{mg} / \mathrm{mL}$.

\section{Conclusões}

O processo redox, apresentado pelo eletrodo de pasta de grafite modificado com nitroprussiato de ferro, $\mathrm{Fe}(\mathrm{II}) \mathrm{NP}\left(\mathrm{E}_{\frac{1}{2}}^{0 \mathrm{x}}\right)_{1}=0.24 \mathrm{~V}$ apresenta atividade eletrocatalítica para a oxidação de ácido ascórbico $(180 \mathrm{mV})$. A faixa linear obtida para a determinação do ácido ascórbico foi de $1.0 \times 10^{-3}$ a $2.6 \times 10^{-2} \mathrm{~mol} \mathrm{~L}^{-1}$ apresentando um limite de detecção de $1.4 \times 10^{-3} \mathrm{~mol} \mathrm{~L}^{-1}$ e sensibilidade amperométrica de $18 \mathrm{~mA} / \mathrm{mol} \mathrm{L}^{-1}$.

Como aplicação direta do eletrodo de pasta de grafite modificado com FE(II)NP pode-se determinar ácido ascórbico em um fármaco (Cewin). Quando comparada a outros métodos eletroanalíticos, a principal vantagem do eletrodo modificado com FE(II)NP, é que o mesmo é de fácil confecção e permite que sua superfície seja renovável. Esta característica é importante quando se quer executar efetivamente várias medidas em curto espaço de tempo; uma outra vantagem é a falta de necessidade de qualquer tratamento químico prévio.

\section{Referências}

1. K. Kalcher, J.M. Kanffmann, J. Wang, J. Scancara, K. Vytras, C. Neuhold, Z.Yang, Electroanalysis 7 (1995) 5.

2. R. Garjonytê, A. Malinauskas, Sensors and Actuators B 46 (1998) 236.

3. D.R. Shakaran, S.S. Narayanan, Bull. Electrochem. 4 (1998) 267.

4. S.M. Chen, J. Electroanal. Chem. 521 (2002) 29.

5. I.L. De Mattos, L. Gorton, Quimica Nova 24 (2001) 200. 
6. N.R. De Toccani, K. Rajeshwar, R.O. Lesna, Electrochimica Acta 45 (2000) 3403.

7. X.P. Cui, L. Hong, X.Q. Lin, Anal. Sci. 18 (2002) 543.

8. H. Razmi-Nerbin, M.H. Pournaghi-Azar, J. Solid State Electrochemistry 6 (2002) 126.

9. T.J. Maflate, T. Nyokong, J. Electroanal. Chem. 408 (1996) 213-218.

10. V.D. Ivanov, M.M. Kaplum, Russian J. Electrochem. 14 (1998) 559.

11. D.R. Carmo, R.M. Silva, N.R. Stradiotto, Eclética Química, in press.

12. S.P. Arya, M. Mahajan, P. Jaim, Anal. Chim. Acta. 417 (2000) 1.

13. S.P. Arya, M. Mahajan, P. Jaim, Anal. Sci. 14 (1998) 889.

14. J. Augustm, C. Beck, G.I. Marousck, J. Food Sci. 46 (1981) 312.

15. N.K. Faudey, Anal. Chem. 34 (1982) 793.

16. J.M. Kim, Y. Huang, R.D. Shimid, Anal. Lett. 23 (1990) 2275.

17. A.V. Ferena, O. Filho-Fatibello, Anal. Chim. Acta 366 (1998) 55.

18. M.H. Bur-Nguyen, J. Chromatogr. 196 (1980) 163.

19. A.G. Fogg, A.M. Summan, Analyst 108 (1983) 691.

20. R. Sandulescu, R. Obrean, L. Roman, Farmacia 45 (1997) 23.

21. British Pharmacopoeia, $5^{\text {th }}$ Ed., HM Stationery Office, London, 1998. 